NSF-ITP-93-68

TPI-MINN-93/33-T

UMN-TH-1208/93

July 1993

\title{
Differential Distributions in Semileptonic Decays of the Heavy Flavors in QCD
}

\author{
B. Blok日 \\ Institute for Theoretical Physics \\ University of California, Santa Barbara, CA 93106 \\ and \\ L. Koyrakh [, M. Shifman [5 and A.I. Vainshtein t田 \\ Theoretical Physics Institute \\ University of Minnesota, Minneapolis, MN 55455
}

\begin{abstract}
A generalization of the operator product expansion is used to find the differential distributions in the inclusive semileptonic weak decays of heavy flavors in QCD. In particular, the double distribution in electron energy and invariant mass of the lepton pair is calculated. We are able to calculate the distributions in essentially model-independent way as a series in $m_{Q}^{-1}$ where $m_{Q}$ is the heavy quark mass. All effects up to $m_{Q}^{-2}$ are included.
\end{abstract}

\footnotetext{
${ }^{1}$ e-mail address: BLOK@SBITP.BITNET

${ }^{2}$ e-mail address: LEVKOYRAKH@PHYSICS.SPA.UMN.EDU

${ }^{3}$ e-mail address: SHIFMAN@UMNACVX

${ }^{4}$ and Budker Institute of Nuclear Physics, Novosibirsk 630090, Russia

${ }^{5}$ e-mail address: VAINSHTE@UMNACVX
} 


\section{Introduction}

Differential distributions in semileptonic decays of heavy flavors are used for measurements of the CKM matrix elements, key phenomenological parameters of the standard model. To extract the CKM matrix elements from data one needs to disentangle the effects of strong interactions at large distances from the quark-lepton lagrangian known at short distances.

Up to now essentially two approaches are applied to describe nonperturbative strong interaction effects in the inclusive weak decays: the naive parton model amended to include the motion of the heavy quark inside the decaying meson [1]; and the 'exclusive variant' based on summation of different channels, one by one [2]. Both approaches are admittedly model-dependent, neither their accuracy nor the connection to the fundamental parameters of QCD are clear a priori. Each of them needs an input from constituent quark model to parametrize nonperturbative effects. The latter play an especially important role in the form of the spectra near the endpoints.

The need for the model-independent QCD-based predictions is apparent. Considerable progress achieved recently in the theory of preasymptotic effects (proportional to powers of $1 / m_{Q}$ where $m_{Q}$ is the heavy quark mass) allows one to make these predictions.

The theoretical construction presented in this paper is, in a sense, a generalization and combination of the formalisms which are used in deep inelastic scattering and total cross section of $e^{+} e^{-}$annihilation. The expansion parameter in deep inelastic scattering is $Q^{-1}$ where $Q$ is the momentum transfer. In the problem at hand the expansion parameter is $m_{Q}^{-1}$ or, more exactly, the inverse energy released in the final hadronic state (in the rest frame of the decaying quark).

In the classical problems of this type, like $e^{+} e^{-}-$annihilation, there are two alternative ways to get predictions. The first approach having a solid theoretical justification in terms of OPE [4] is based on calculations in the euclidean domain where one can apply OPE. The contact with the observable quantities is made through the dispersion relations and in this way predictions for certain integrals are obtained. In the second approach we perform the calculations directly in Minkowski domain. Although formally this calculation refers to large distances from the first approach we know that in specific integrals large distance contributions drop out. Therefore the results obtained in this way, although not valid literally, should be understood in 
the sense of duality: being smeared over some duality interval the theoretical prediction should coincide with the smeared experimental curve. The inclusive weak decays will be treated within the second approach. The averaging mainly refers to the invariant mass of the inclusive hadronic state produced in the decay considered.

If the invariant mass of the final hadronic state is large this is not a constraint at all since the theory 'itself' takes care of the averaging required by duality. In the opposite limit, near a spectral endpoint, the smearing is not provided for free. We will discuss the issue in some detail below.

Although we explicitly work in the Minkowski kinematics we always keep in mind the relationship to the euclidean domain and the corresponding operator product expansion. The first analysis of this type has been outlined in [3] for inclusive heavy flavor decay rates. A general analysis of the semileptonic inclusive spectra along this line is presented in ref. [9]. In that work it was observed, in particular, that the leading operator and those appearing at the next-to-leading order have a gap in dimensions of two units, and, consequently, the $\mathcal{O}\left(m_{Q}^{-1}\right)$ term should be absent in certain quantities. The analysis presented in [9] was not backed up, however, by concrete calculations of the preasymptotic effects. Recently this formalism has been systematically developed and applied to the non-leptonic decays of heavy flavors [5, 6] and the charged-lepton energy spectrum in the semileptonic decays [7] (see also [8]). The present work is a natural continuation of ref. [7].

We generalize the results of ref. [7] to find the complete inclusive distributions in the semileptonic decays. The leptonic variables $-E_{e}, q^{2}$ and $q_{0}$, where $E_{e}$ is the charged lepton energy and $q$ is the momentum of the lepton pair [ - are kept fixed which automatically fixes the invariant mass of the inclusive hadronic state. Integrating over $q_{0}$ we obtain the double spectral distribution in $E_{e}$ and $q^{2}$.

At the first stage we construct the transition operator $T(Q \rightarrow X \rightarrow Q)$ describing the forward scattering amplitude of the heavy quark $Q$ on a weak current. Our focus is the influence of the 'soft' modes (background fields) on the transition operator $T_{\mu \nu}$ which is expressed as an infinite series in the local operators built from the gluon and/or light-quark fields bilinear in $Q \bar{Q}$.

The local operators are ordered according to their dimensions; the coefficient functions contain the corresponding powers of $1 / m_{Q}$ (or $1 / E$, the

\footnotetext{
${ }^{6}$ The charged lepton produced will be generically called 'electron' hereafter.
} 
energy release). At sufficiently large $m_{Q}$ or $E$ the operators with the lowest dimensions dominate, and the infinite series can be truncated.

At the next stage the matrix elements of the relevant operators over the initial heavy hadron $H_{Q}$ must be evaluated. Unfortunately, in the presentday QCD the matrix elements over the hadronic states are not theoretically calculable. In some instances they can be related, through heavy quark symmetries, to measurable quantities [12], [2]; in other cases they have to be parametrized. These parameters play the role analogous to the gluon condensate [10]. As a matter of fact, at the level of the leading preasymptotic corrections only two operators are relevant. The matrix element of the first one can be related to the mass splittings of the vector and pseudoscalar heavy mesons. The matrix element of the second one has the meaning of the average square of the spatial momentum of the heavy quark $Q$ in $H_{Q}$ and the state must be treated as a parameter.

Finally, the observed decay rates and spectra are obtained by taking the discontinuity of the hadronic tensor $<H_{Q}\left|T_{\mu \nu}\right| H_{Q}>$ and convoluting the result with the lepton currents and appropriate kinematic factors.

In this paper we consider the differential distributions in the semileptonic decays at the level of $O\left(m_{Q}^{-2}\right)$. The differential distributions are measured experimentally in the $B$ meson decays and will be used for more precise determination of $V_{u b}$, for example. This was a primary motivation for our investigation. We would like to make it as close to the fundamental QCD as possible.

The organization of the paper is as follows. In Sect. 2 we describe the kinematics and in Sect. 3 we present the operator product expansion. In Sect. 4 we derive the differential distributions. Sect. 5 is devoted to the analysis of our distributions and limitations on the their use. Our results are summarized in Sect. 6. Appendix contains expressions for hadronic invariant functions.

\section{Kinematical analysis}

We will consider the inclusive weak decays of the mesons (or baryons) with the open heavy flavor into the lepton pair plus (inclusive) hadronic state $H_{Q}\left(p_{H}\right) \rightarrow l\left(p_{l}\right)+\bar{\nu}\left(p_{\nu}\right)+$ hadrons. Our final goal is to calculate the differ- 
ential decay rate

$$
\frac{d^{3} \Gamma}{d E_{e} d q^{2} d q_{0}}
$$

where $E_{e}$ is the energy of the emitted electron and $q^{\mu}=p_{l}^{\mu}+p_{\nu}^{\mu}$ is the 4momentum of the lepton pair. In order to find the differential distributions we need to know the amplitude of the process, which is given by the expression

$$
\mathcal{M}=V_{q Q} \frac{G_{F}}{\sqrt{2}} \bar{e} \Gamma_{\nu} \nu<X\left|j_{\nu}\right| H_{Q}>
$$

Here $V_{q Q}$ is the corresponding Cabibbo-Kobayashi-Maskawa matrix element, $j_{\mu}=\bar{q} \Gamma_{\mu} Q$ is the electroweak currents, $\Gamma_{\mu}=\gamma_{\mu}\left(1+\gamma_{5}\right)$. (Although our theory is general we will keep in mind the $b \rightarrow c$ and $b \rightarrow u$ decays, so that $Q=b$ and $q=c$ or $u$ ). The differential distributions we are interested in are given by the modulus squared of the amplitude (21) summed over the final hadronic states.

The modulus squared of the amplitude summed over the final hadronic states can be written as

$$
|\mathcal{M}|^{2}=\left|V_{q Q}\right|^{2} G_{F}^{2} M_{H_{Q}} l^{\mu \nu} W_{\mu \nu}
$$

where $M_{H_{Q}}$ is the mass of hadron $H_{Q}, W_{\mu \nu}$ is the hadronic tensor

$$
W_{\mu \nu}=(2 \pi)^{4} \delta^{4}\left(p_{H_{Q}}-q-p_{X}\right) \sum_{X} \frac{1}{2 M_{H_{Q}}}<H_{Q}\left|j_{\mu}^{\dagger}(0)\right| X><X\left|j_{\nu}(0)\right| H_{Q}>,
$$

and $l^{\mu \nu}$ is the lepton tensor

$$
l^{\mu \nu}=8\left[\left(p_{e}\right)^{\mu}\left(p_{\nu}\right)^{\nu}+\left(p_{e}\right)^{\nu}\left(p_{\nu}\right)^{\mu}-g^{\mu \nu}\left(p_{e} \cdot p_{\nu}\right)+i \epsilon^{\mu \nu \alpha \beta}\left(p_{e}\right)^{\alpha}\left(p_{\nu}\right)^{\beta}\right] .
$$

Let us introduce the hadronic structure functions $w_{i}$ and parametrize the hadronic tensor in the following way:

$$
\begin{array}{r}
W_{\mu \nu}=-w_{1} g_{\mu \nu}+w_{2} v_{\mu} v_{\nu}-i w_{3} \epsilon_{\mu \nu \alpha \beta} v_{\alpha} q_{\beta}+ \\
w_{4} q_{\mu} q_{\nu}+w_{5}\left(q_{\nu} v_{\mu}+q_{\mu} v_{\nu}\right) .
\end{array}
$$

Here $q_{\mu}=\left(p_{e}+p_{\nu}\right)_{\mu}$ is the 4 - momentum of the lepton pair, $v_{\mu}=\left(p_{H_{Q}}\right)_{\mu} / M_{H_{Q}}$ is the 4-velocity of the initial hadron. Note that we have omitted the structure $q_{\mu} v_{\nu}-q_{\nu} v_{\mu}$ which can not appear because of the $T$-invariance. The structure 
functions $w_{i}$ depend on two invariant variables, $q \cdot v$ and $q^{2}$. In the rest frame of $H_{Q}$ which will be used throughout the paper $q \cdot v=q_{0}$, so $w_{i}=w_{i}\left(q_{0}, q^{2}\right)$. The convolution of $W_{\mu \nu}$ with the lepton tensor (5) is given by the expression:

$$
W_{\mu \nu} l^{\mu \nu}=4\left\{2 q^{2} w_{1}+\left[4 E_{e}\left(q_{0}-E_{e}\right)-q^{2}\right] w_{2}+2 q^{2}\left(2 E_{e}-q_{0}\right) w_{3}\right\} .
$$

We see that only three invariant functions are relevant for the processes we are considering in this paper. At this step we encounter the third variable, the electron energy $E_{e}=p_{e} \cdot p_{H_{Q}} / M_{H_{Q}}$, entering through the leptonic tensor.

Finally the formulae for the differential width takes the form

$\frac{d^{3} \Gamma}{d E_{e} d q^{2} d q_{0}}=\left|V_{q Q}\right|^{2} \frac{G_{F}^{2}}{32 \pi^{2}}\left[2 q^{2} w_{1}+\left[4 E_{e}\left(q_{0}-E_{e}\right)-q^{2}\right] w_{2}+2 q^{2}\left(2 E_{e}-q_{0}\right) w_{3}\right]$

This expression concludes the kinematical analysis. Our task is, of course, the calculation of the invariant functions $w_{i}\left(q_{0}, q^{2}\right)$. We will proceed to this calculation in the next section.

\section{Operator product expansion}

In this section we will discuss the derivation of the tensor $W_{\mu \nu}$. The Operator product expansion is similar to that in the deep inelastic scattering. It is convenient to introduce the hadronic tensor $h_{\mu \nu}$ (forward scattering amplitude)

$$
h_{\mu \nu}=i \int d^{4} x e^{-i q x} \frac{1}{2 M_{H_{Q}}}<H_{Q}\left|T\left\{j_{\mu}^{+}(x) j_{\nu}(0)\right\}\right| H_{Q}>.
$$

The absorptive part of this tensor reduces to $W_{\mu \nu}$ discussed above

$$
W_{\mu \nu}=(1 / i) \operatorname{disc}\left(h_{\mu \nu}\right) .
$$

Here $\operatorname{disc}\left(h_{\mu \nu}\right)$ is the discontinuity of the forward scattering amplitude $h_{\mu \nu}$ on the physical cut in the complex plane of the variable $q_{0}$. Of course, $h_{\mu \nu}$ can be expanded into the same set of structures as $W_{\mu \nu}$ (see. eq. (6))

$$
h_{\mu \nu}=-h_{1} g_{\mu \nu}+h_{2} v_{\mu} v_{\nu}-i h_{3} \epsilon_{\mu \nu \alpha \beta} v_{\alpha} q_{\beta}+h_{4} q_{\mu} q_{\nu}+h_{5}\left(q_{\nu} v_{\mu}+q_{\mu} v_{\nu}\right) \text {, }
$$

and the relation (10) implies that

$$
w_{i}=2 \operatorname{Im}\left(h_{i}\right)
$$



$T_{\mu \nu}$

Let us remind that $h_{\mu \nu}$ is the matrix element of the transition operator

$$
\begin{gathered}
h_{\mu \nu}=\frac{1}{2 M_{H_{Q}}}<H_{Q}\left|T_{\mu \nu}\right| H_{Q}>, \\
T_{\mu \nu}=i \int d^{4} x e^{-i q x} T\left\{j_{\mu}^{+}(x) j_{\nu}(0)\right\},
\end{gathered}
$$

so below we will construct OPE for the product of currents in equation (14). Having in mind the relationship to euclidean analysis discussed above we will treat our expansion in the same way as a normal euclidean OPE. In the asymptotic limit $m_{Q} \rightarrow \infty$ the hadronic tensor $h_{\mu \nu}$ is given by the tree graph of Fig. 1. This graph defines the matrix element of the transition operator $T_{\mu \nu}$ over the heavy quark state,

$$
<Q\left|T_{\mu \nu}\right| Q>=-\bar{u}_{Q} \Gamma_{\mu} \frac{1}{\not P-\not \subset-m_{q}} \Gamma_{\nu} u_{Q}
$$

This expression represents nothing else but the free quark decay. In the asymptotic regime $m_{Q} \rightarrow \infty$ the interaction of the heavy quark with the gluon/light quark medium, as well as its intrinsic notion inside the hadron can be neglected. Then

$$
P_{\mu}=P_{0 \mu} \equiv m_{Q} v_{\mu}
$$

where $v_{\mu}$ is the $H_{Q}$ 4-velocity.

Equation (15) allows one to immediately write down the operator form in the approximation at hand (only operators bilinear in $Q, \bar{Q}$ are considered, see a discussion of other operators at the end of the section):

$$
\begin{aligned}
T_{\mu \nu}= & -\bar{Q} \Gamma_{\mu} \frac{1}{k /-m_{q}} \Gamma_{\nu} Q= \\
& -\frac{2}{\left(k^{2}-m_{q}^{2}\right)}\left[g_{\alpha \mu} k_{\nu}+g_{\alpha \nu} k_{\mu}-g_{\mu \nu} k_{\alpha}-i \epsilon_{\mu \nu \alpha \beta} k_{\beta}\right] \bar{Q} \gamma^{\alpha}\left(1+\gamma_{5}\right) Q
\end{aligned}
$$

where $k=P_{0}-q$.

As we see, the two operators $\bar{Q} \gamma_{\alpha} \bar{Q}$ and $\bar{Q} \gamma_{\alpha} \gamma_{5} \bar{Q}$ showed up in the operator expansion at the level considered. Note, that the $\bar{Q} \gamma_{\alpha} \gamma_{5} \bar{Q}$ term vanishes after averaging over the unpolarized hadronic states.

In this paper the perturbative corrections in $\alpha_{s}$ are not touched upon at all. As for nonperturbative corrections they appear due to interactions with 
the soft medium of the light cloud in $H_{Q}$. By taking these interactions into account we isolate two types of effects. First, the fast quark $q$ produced does not propagate as a free one, but interacts with the background fields; these corrections will be included explicitly. Second, the heavy quark $Q$ also does not live in the empty space; it is surrounded by the light cloud. In particular, due to this fact the heavy quark momentum does not coincide with $m_{Q} v_{\mu}$. This large distance effect will not be calculated explicitly, but implicitly it will be reflected in the $H_{Q}$ matrix elements of the operators in $T_{\mu \nu}$. This is in full analogy with what people usually do in the deep inelastic scattering. The influence of the background field on the transition operator is summarized by the following expression

$$
T_{\mu \nu}=-\int d x e^{-i q x} \bar{Q}(x) \Gamma_{\mu} S_{q}(x, 0) \Gamma_{\nu} Q(0)
$$

where $S_{q}(x, 0)$ is the propagator of the quark $q$ in an external gluon field $A_{\mu}^{a}$. It is convenient to use the Schwinger technique of treating the motion in an external field (for a review of QCD adaptation see, e.g. ref. [13]). Within that formalism the propagator $S_{q}$ is presented by the following expression

$$
S_{q}(x, 0)=\left(x\left|\frac{1}{\not \supset-m_{q}}\right| 0\right)
$$

Here $\mathcal{P}=\gamma^{\mu}\left(p_{\mu}+A_{\mu}(X)\right), A_{\mu}=g A_{\mu}^{a} T^{a}$ is a gluon field in the matrix representation. Furthermore, the operator of coordinate $X_{\mu}$ and momentum $p_{\mu}$ are introduced, (thus the field $A_{\mu}(X)$ becomes an operator function of $X_{\mu}$ ), with the commutation relations

$$
\left[p_{\mu}, X_{\nu}\right]=i g_{\mu \nu}, \quad\left[X_{\mu}, X_{\nu}\right]=0, \quad\left[p_{\mu}, p_{\nu}\right]=0 .
$$

The states $\mid x)$ are the eigenstates of the operator $\left.\left.X_{\mu}, X_{\mu} \mid x\right)=x_{\mu} \mid x\right)$.

Combining equations (19) and (18), we arrive at

$$
T_{\mu \nu}=-\int d x e^{-i q x}\left(x\left|\bar{Q}(x) \Gamma_{\mu} \frac{1}{\mathcal{P}-m_{q}} \Gamma_{\nu} Q(X)\right| 0\right) .
$$

As we have discussed above the operator $\mathcal{P}_{\mu}$ contains a large mechanical part $\left(P_{0}\right)_{\mu}=m_{Q} v_{\mu}$; the deviation from $P_{0}$ will be separated explicitly

$$
\mathcal{P}_{\mu}=\left(P_{0}\right)_{\mu}+\pi_{\mu}
$$


and we will expand in $\pi_{\mu}$. In this paper we will limit ourselves to the terms up to $\mathcal{O}\left(\pi^{2}\right)$ corresponding to $1 / m_{Q}^{2}$ corrections. The master formulae to perform the expansion is

$$
T_{\mu \nu}=-\int d x\left(x\left|\bar{Q}(X) \Gamma_{\mu} \frac{1}{\not P_{0}-q-m_{q}+\not \not \mid} \Gamma_{\nu} Q(X)\right| 0\right) .
$$

There is a subtle point in the description of the formalism given above. Technically in the computation the $A_{\mu}(x)$ is assumed to be a c-number background field while in the final expression for local operators it should be understood as a second quantized operator. Since we are not considering any loop corrections this substitution is justified.

Let us now discuss the set of the operators relevant to the order $\mathcal{O}\left(m_{Q}^{-2}\right)$. Without loss of generality we can work in the rest frame of the hadron $H_{Q}$, i.e. $v_{\mu}=(1,0,0,0)$. Only those operators will be retained which produce non-vanishing results after being averaged over $H_{Q}$. The leading operator, as it was discussed above, is

$$
\bar{Q} \gamma_{0} Q \text {, }
$$

it's matrix element is fixed by the vector current conservation to be

$$
\frac{1}{2 M_{H_{Q}}}<H_{Q}\left|\bar{Q} \gamma_{0} Q\right| H_{Q}>=1,
$$

Equation (25) is given in relativistic normalization we are using throughout this paper. In the non-relativistic normalization there is no need in the factor $1 / 2 M_{H_{Q}}$ in the l.h.s.

As it has been noted in the ref. 90 there are no operators of dimension 4 in the problem at hand. The set includes two operators of dimension 5:

$$
\begin{gathered}
\mathcal{O}_{G}=\frac{i}{2} \bar{Q} \sigma^{\alpha \beta} G_{\alpha \beta} Q, \\
\mathcal{O}_{\pi}=-\bar{Q} \vec{D}^{2} Q=\bar{Q} \vec{\pi}^{2} Q,
\end{gathered}
$$

where $\sigma^{\alpha \beta}=\frac{1}{2}\left(\gamma^{\alpha} \gamma^{\beta}-\gamma^{\beta} \gamma^{\alpha}\right)$, and $G_{\alpha \beta}=g G_{\alpha \beta}^{q} T^{a}$ is the gluon field strength tensor. The classification above takes into account the fact that the quark field $Q$ satisfies the equation of motion. In particular, it stems that the 
operator $\bar{Q} Q$ is not independent but is reducible to three operators (24), (26) and (27):

$$
\bar{Q} Q=\bar{Q} \gamma_{0} Q-\frac{1}{2 m_{Q}^{2}} \bar{Q} \vec{\pi}^{2} Q+\frac{i}{4 m_{Q}^{2}} \bar{Q} \sigma^{\alpha \beta} G_{\alpha \beta} Q+\mathcal{O}\left(m_{Q}^{-3}\right) .
$$

To get eq. (28) we observe that the lower component of $Q$ is related to the upper one in the following way

$$
\frac{1-\gamma_{0}}{2} Q=\frac{1}{2 m_{Q}} \vec{\pi} \vec{\sigma} \frac{1+\gamma_{0}}{2} Q+\mathcal{O}\left(m_{Q}^{-2}\right)
$$

and the difference between $\bar{Q} Q$ and $\bar{Q} \gamma_{0} Q$ is due to the product of the lower components. (Here and below we will stick to the $H_{Q}$ rest frame.)

A few other useful relations which can be obtained in the same manner and are valid at the level $\mathcal{O}\left(m_{Q}^{-2}\right)$ are:

$$
\begin{aligned}
& \bar{Q} \vec{\gamma} \vec{\pi} Q= \frac{1}{m_{Q}} \bar{Q}\left(\vec{\pi}^{2}-\frac{i}{2} \sigma G\right) Q+\mathcal{O}\left(m_{Q}^{-2}\right), \\
& \bar{Q} \vec{\gamma} \vec{\pi} \gamma_{0} Q=\mathcal{O}\left(m_{Q}^{-2}\right), \\
& \bar{Q} \pi_{0} Q=\frac{1}{2 m_{Q}} \bar{Q}\left(\vec{\pi}^{2}-\frac{i}{2} \sigma G\right) Q+\mathcal{O}\left(m_{Q}^{-2}\right) .
\end{aligned}
$$

A few comments are in order here concerning the actual technique of constructing the OPE. Since we work in the $H_{Q}$ rest frame it is convenient to compute different components of $T_{\mu \nu}$ separately, $T_{00}, T_{0 i}, T_{i 0}$ and $T_{i j}$. The calculation itself is a straightforward although rather tedious procedure of expanding the denominator in eq. (23) in $\not t$ using the properties of the $\gamma$ matrices, the commutation relation

$$
\left[\pi_{\mu}, \pi_{\nu}\right]=i G_{\mu \nu}
$$

and equations (30) - (32).

Notice that we must keep the terms of the first order in $\pi_{0}$ and of the second order in $\vec{\pi}$, since

$$
\pi_{0} Q=\frac{(\vec{\sigma} \vec{\pi})^{2}}{2 m_{Q}} Q+\mathcal{O}\left(m_{Q}^{-2}\right)
$$


Next, observe that the Green function in the background field can be written as follows:

$$
\frac{1}{\not \mathcal{P}-\not q-m_{q}}=\left(\not{P}-\not q+m_{q}\right) \frac{1}{(\mathcal{P}-q)^{2}+(i / 2) \sigma G-m_{q}^{2}} \equiv\left(\not{P}-\not q+m_{q}\right) \frac{1}{\Pi} .
$$

To transpose $1 / \Pi$ with $\Gamma_{\nu}$ it is convenient to use the identity

$$
\begin{aligned}
& \frac{1}{\Pi} \Gamma_{\nu}=\Gamma_{\nu} \frac{1}{\Pi}+\frac{1}{\Pi}\left[\Gamma_{\nu}, \Pi\right] \frac{1}{\Pi} \\
& \quad=\Gamma_{\nu} \frac{1}{\Pi}+\frac{1}{\Pi}\left[\Gamma_{\nu}, \frac{i}{2} \sigma G\right] \frac{1}{\Pi} .
\end{aligned}
$$

Acting on $Q$ and using the equations of motion we can now substitute $1 / \Pi$ in both terms on the right-hand side by

$$
\frac{1}{m_{Q}^{2}-m_{q}^{2}-2 \mathcal{P} q+q^{2}}
$$

provided that we limit ourselves to terms up to $\mathcal{O}\left(m_{Q}^{-2}\right)$. The second term in (36) can be simplified even further since here we can additionally neglect $\pi$ in $\mathcal{P}=P_{0}+\pi$.

We split the calculation into three parts: Vector $\times$ Vector, Axial $\times$ Axial and Vector $\times$ Axial in correspondence with the structure of $\Gamma_{\mu}$ as a sum of vector and axial vector, $\Gamma_{\mu}=\gamma_{\mu}+\gamma_{\mu} \gamma_{5}$. The full hadronic tensor $h_{\mu \nu}$ is given then by the following expression:

$h_{\mu \nu}=h_{\mu \nu}^{V V}+h_{\mu \nu}^{A A}+h_{\mu \nu}^{A V}+h_{\mu \nu}^{V A}=\frac{1}{2 M_{H_{Q}}}<H_{Q}\left|T_{\mu \nu}^{V V}+T_{\mu \nu}^{A A}+T_{\mu \nu}^{A V}+T_{\mu \nu}^{V A}\right| H_{Q}>$.

The complete expressions for the hadronic invariant functions are given in the Appendix. In the order $\mathcal{O}\left(m_{Q}^{-2}\right)$ they are defined by matrix elements of operators $\mathcal{O}_{G}, \mathcal{O}_{\pi}$ given by eqs. (26), (27):

$$
\begin{gathered}
\frac{1}{2 M_{H_{Q}}}<H_{Q}\left|\bar{Q} \frac{i}{2} \sigma_{\mu \nu} G^{\mu \nu} Q\right| H_{Q}>=\mu_{G}^{2}, \\
\frac{1}{2 M_{H_{Q}}}<H_{Q}\left|\bar{Q} \vec{\pi}^{2} Q\right| H_{Q}>=\mu_{\pi}^{2},
\end{gathered}
$$


The parameter $\mu_{G}^{2}$ coincides with $m_{\sigma H}^{2}$ introduced in 14]. For mesonic states it is expressible in terms of the quantity measured experimentally - the hyperfine mass splittings, and it has the zero value for baryonic states of the type of $\Lambda_{Q} ; \mu_{\pi}^{2}$ has the meaning of the average square of spatial momentum of the heavy quark $Q$ in the hadronic state $H_{Q}$. The two parameters, $\mu_{G}^{2}$ and $\mu_{\pi}^{2}$, often appear in the combination $\mu_{\pi}^{2}-\mu_{G}^{2}$, cf. equation (28).

The last comment of this Section is about the operators which are not bilinear in $\bar{Q}, Q$ fields. The simplest example of appearance of such operators is given by the diagram of Fig.2 where the heavy quark $Q$ propagates between the current vertices. This diagram is similar to the one of Fig.1, and the corresponding operator follows from eq.(18) by substitution $\bar{Q}, Q \Rightarrow \bar{q}, q$, $m_{q} \Rightarrow m_{Q}, k_{\mu} \Rightarrow q_{\mu}$. The additional term in $T_{\mu \nu}$ has the form

$$
\begin{aligned}
\Delta T_{\mu \nu}= & -\bar{q} \Gamma_{\nu} \frac{1}{q-m_{Q}} \Gamma_{\mu} q= \\
& -\frac{2}{\left(q^{2}-m_{Q}^{2}\right)}\left[g_{\alpha \mu} q_{\nu}+g_{\alpha \nu} q_{\mu}-g_{\mu \nu} q_{\alpha}-i \epsilon_{\mu \nu \alpha \beta} q_{\beta}\right] \bar{q} \gamma^{\alpha}\left(1+\gamma_{5}\right) q
\end{aligned}
$$

Matrix element of the operator $\bar{q} \gamma^{\alpha} q$ over $H_{Q}$ state counts the number of quarks $q$ and is not small in general. The operator coefficient given by eq. (42) is particularly large when $q^{2} \rightarrow m_{Q}^{2}$.

In terms of the intermediate hadronic states in the forward scattering off $H_{Q}$ this contribution is due to states in crossing channel containing two $Q$ quarks - the corresponding problem was pointed out in ref.[9]. The crosschannel is not related to the weak inclusive decays under consideration and the analysis above shows that we can consistently omit the crossing channel together with operators in $T_{\mu \nu}$ related to this channel. Similar consideration can be carried out for other operators containing no heavy quark fields $Q, \bar{Q}$.

\section{Calculation of the differential distributions}

The differential distributions we are interested in are determined by equation (8) containing three invariant functions $w_{1}, w_{2}$ and $w_{3}$. They are obtained from the results for $h_{i}$ (see Appendix) by taking the imaginary parts of the corresponding functions (see. eq. (12)). The imaginary parts are due to the 
poles of $h_{i}$ and are obtained through the relations:

$$
\operatorname{Im} \frac{1}{z^{n}}=\pi \frac{(-1)^{n-1}}{(n-1) !} \frac{d^{n-1}}{d z^{n-1}} \delta(z)
$$

where $z$ is given by

$$
z=m_{Q}^{2}-2 m_{Q} q_{0}+q^{2}-m_{c}^{2} .
$$

We don't present here the expression for the triple differential distribution which can be easily obtained by combining the equations (8), (12), (42) and expressions for $h_{i}$ from the Appendix.

Although the result is written for the physical quantity $d^{3} \Gamma / d E_{e} d q^{2} d q_{0}$, it can not be directly compared with the experimental data. An obvious signal for this is the presence of the delta function and it's derivatives. It is not surprising because we are sitting now right on the physical cut on mass-shell of the q-quark. As we discussed in the introduction our results should be understood in the sense of duality: that is that the predictions should be smeared over certain duality interval. At the moment we have no purely theoretical tools to fix the size of the duality interval, therefore we are forced to rely on qualitative arguments and experimental data. For example the duality interval for $q_{0}$ can be inferred from the distribution in the invariant mass of the final hadronic states. Our $\delta$-functions reflect the resonance structure at low invariant masses. The smearing interval should be chosen in such a way as to cover the entire resonance domain up to the onset of the smooth behavior. Instead of smearing of the distribution one can calculate the average characteristics like the total width $\Gamma$ or $\left\langle M_{X}^{n}\right\rangle$, where $M_{X}$ is the invariant mass of the final hadronic states. The power corrections we have calculated will enter in a specific way in each particular quantity.

Now let us proceed to the calculation of the double differential distribution $d^{2} \Gamma / d E_{e} d q^{2}$. To this end we must integrate over $q_{0}$, rather simple exercise with $\delta$-functions. However if one would perform the integration by merely substitution

$$
q_{0} \rightarrow q_{0}^{*}=\frac{m_{Q}^{2}+q^{2}-m_{q}^{2}}{2 m_{Q}},
$$

and taking the derivatives in the case of $\delta^{\prime}$ and $\delta^{\prime \prime}$, one would get the wrong answer. The point is that integration domain in $q_{0}$ has a boundary from 
below

$$
q_{0} \geq E_{e}+\frac{q^{2}}{4 E_{e}},
$$

which corresponds to $4 E_{e} E_{\nu} \geq q^{2}$. Therefore one should take into account the fact that $q_{0}$ can not cross the boundary (45). For that we introduce $\theta\left(q_{0}-E_{e}-q^{2} / 4 E_{e}\right)$ into the integrand. The occurrence of the $\theta$-function is important for the integration of $\delta^{\prime}\left(q_{0}-q_{0}^{*}\right)$ and $\delta^{\prime \prime}\left(q_{0}-q_{0}^{*}\right)$ which leads to appearance of $\delta\left(q_{0}^{*}-E_{e}-q^{2} / 4 E_{e}\right)$ and $\delta^{\prime}\left(q_{0}^{*}-E_{e}-q^{2} / 4 E_{e}\right)$ in the double distribution $d^{2} \Gamma / d q^{2} d E_{e}$, because of differentiation of the $\theta$-function . The final formulae for the double differential distribution in the lepton energy $E_{e}$ and $q^{2}$ takes the form:

$$
\begin{aligned}
\frac{d^{2} \Gamma}{d x d t} & =\left|V_{q Q}\right|^{2} \frac{G_{F}^{2} m_{Q}^{5}}{96 \pi^{3}} x^{2}\{6(1-t)(1-\rho-x+x t)+ \\
& G_{Q}\left[1-5 \rho+2 t+10 \rho t+10 x t-10 x t^{2}-\right. \\
& \left(-1+6 \rho-5 \rho^{2}+x-5 \rho x+t-2 \rho t+5 \rho^{2} t+x t+15 \rho x t+\right. \\
& \left.\left.5 x^{2} t-2 x t^{2}-10 \rho x t^{2}-10 x^{2} t^{2}+5 x^{2} t^{3}\right) \delta((1-t)(1-x)-\rho)\right]+ \\
& K_{Q}\left[-3+3 \rho+4 t-4 \rho t-6 x t+4 x t^{2}-\right. \\
& \left(1-2 \rho+\rho^{2}-3 x+3 \rho x-3 t+2 \rho t+\rho^{2} t+11 x t-3 \rho x t-\right. \\
& \left.3 x^{2} t-6 x t^{2}-2 \rho x t^{2}+2 x^{2} t^{2}+x^{2} t^{3}\right) \delta((1-t)(1-x)-\rho)+ \\
& (1-\rho-x+x t)(1-t)(1-2 \rho+ \\
& \left.\left.\left.\rho^{2}-2 x t-2 \rho x t+x^{2} t^{2}\right) \delta^{\prime}((1-t)(1-x)-\rho)\right]\right\} .
\end{aligned}
$$

Here we have introduced the dimensionless variables

$$
x=2 E_{e} / m_{Q}, \quad t=q^{2} / 2 m_{Q} E_{e},
$$

and the parameters

$$
\rho=m_{q}^{2} / m_{Q}^{2}, \quad G_{Q}=\mu_{G}^{2} / m_{Q}^{2}, \quad K_{Q}=\mu_{\pi}^{2} / m_{Q}^{2}
$$

Let us emphasize that the scale $m_{Q}$ used in equation (47) is the heavy quark mass and does not coincide with $M_{H_{Q}}$ which is normally used in the experimental distributions.

The fact that OPE generates corrections only of the order of $\mathcal{O}\left(m_{Q}^{-2}\right)$ (terms proportional to $K_{Q}$ and $G_{Q}$ ) is valid for the distributions only if we use $m_{Q}$ as a scale, i.e. in the variables $x, t$. Of course one can easily rescale 
them to $M_{H_{Q}}$; then the corrections of the order of $\mathcal{O}\left(m_{Q}^{-1}\right)$ will show up for trivial kinematical reasons.

We can proceed further and obtain the energy spectrum by integrating over $q^{2}$. The range of integration is given by

$$
0 \leq t \leq 1-\frac{\rho}{1-x}
$$

The result for the energy spectrum coincides with that obtained in [7]. For the sake of completeness we present it here I:

$$
\begin{aligned}
\frac{d \Gamma}{d x}= & \left|V_{q Q}\right|^{2} \frac{G_{F} m_{Q}^{5}}{192 \pi^{3}} \theta(1-x-\rho) 2 x^{2}\left\{(1-f)^{2}(1+2 f)(2-x)+(1-f)^{3}(1-x)+\right. \\
& (1-f)\left[(1-f)\left(2+\frac{5}{3} x-2 f+\frac{10}{3} f x\right)-\frac{f^{2}}{\rho}\left(2 x+f\left(12-12 x+5 x^{2}\right)\right)\right] G_{Q}- \\
& {\left[\frac{5}{3}(1-f)^{2}(1+2 f) x+\frac{f^{3}}{\rho}(1-f)\left(10 x-8 x^{2}\right)+\right.} \\
& \left.\left.\frac{f^{4}}{\rho^{2}}(3-4 f)\left(2 x^{2}-x^{3}\right)\right] K_{Q}\right\},
\end{aligned}
$$

where $f=\rho /(1-x)$. Finally, performing the last integration over $x$ in the domain

$$
0 \leq x \leq 1-\rho
$$

we arrive to the total width coinciding with that in [8]:

$$
\Gamma=\left|V_{q Q}\right|^{2} \frac{G_{F} m_{Q}^{5}}{192 \pi^{3}}\left[z_{0}\left(1+\frac{1}{2}\left(G_{Q}-K_{Q}\right)\right)-2 z_{1} G_{Q}\right]
$$

where $z_{0}=1-8 \rho+8 \rho^{3}-\rho^{4}-12 \rho^{2} \log \rho$ and $z_{1}=(1-\rho)^{4}$.

Now let us discuss the characteristic features of the double distribution (46). The most striking one is the presence of the singular terms. The technical reason for occurrence of those terms was that we expanded the denominator of the pole expression (23) in $\pi$ and $\sigma G$. Physically this expansion reflects the shifts of the masses of particles due to the nonperturbative effects. As it was mentioned above these singularities reflect the structure of the resonance domain and the predictions suitable for comparison with

\footnotetext{
${ }^{7}$ Let us draw the reader's attention to the difference of notation: $y$ in $[7$ is equal to our $x$.
} 
the experimental data require smearing over corresponding interval. To illustrate the most salient features of our prediction let us concentrate on the physically interesting case of the $b \rightarrow u$ transition.

For massless $u$ quark the kinematical region of $b$ quark semileptonic decay is shown on Fig.3. It has the form of a square with the side equal to 1 in the plane $\left(x=2 E_{e} / m_{b}, t=q^{2} / 2 m_{b} E_{e}\right)$. The right-hand side of the square corresponds to the maximal energy of electron $E_{e}=m_{b} / 2$ while the upper side is a maximal energy of neutrino. In the real $B$ meson decay the kinematical region is certainly wider; if one neglects the pion mass the region is the square with the side $x_{\max }=t_{\max }=M_{B} / m_{b}$. The origin of this window is related to the motion of the heavy $b$ quark inside of $B$ meson. In our calculations we account for nonzero momentum of the $b$ quark in the form of expansion which produced singular $\delta$ and $\delta^{\prime}$ terms on the boundary. It is possible to show (see refs.[0], [18]) that the expansion breaks down at distances $\sim\left(M_{B}-m_{b}\right) / m_{b}$ near the boundary, so we need to average our results over the range of the order of the window between quark and hadron boundaries. It is interesting to note that the distribution spread off the distances of the order $\left(M_{B}-m_{b}\right) / m_{b}$ while the corrections to integrals are only of the second order in $1 / m_{b}$.

Another effect we need to account for is the structure of resonance region near the low end of the hadronic invariant masses. To imitate the effect let us imagine that this region corresponds to the $u$ quark fragmentation into the hadronic states with $s$ (the square of the invariant mass) from $s=0$ to $s=s_{0}=2 \mathrm{GeV}^{2}$. The curve corresponding to $s=s_{0}$ at Fig. 3 is given by the equation:

$$
(1-t)(1-x)=s_{0} / m_{b}^{2}
$$

and the resonance region should be included as a whole into the process of averaging; we can predict the integral but not the structure.

\section{Application to the analysis of the experi- mental data}

In order to compare our results with the experimental data we use the following values of parameters in eq. (46). First, we use $m_{b} \sim 4.8 \mathrm{GeV}$ as deduced from QCD analyses of the Ypsilon system and $M_{B} \sim 5.3 \mathrm{GeV}$. For 
the parameter $G_{b}$ we use the value 14

$$
G_{b}=\frac{3}{4}\left(M^{2}\left(B^{*}\right)-M^{2}(B)\right) /\left(4 m_{b}^{2}\right) \sim 0.017
$$

As a representative value we use for the parameter $K_{b}$ the value $\sim 0.02$ (see ref. [15]-[17]).

To construct the quantity convenient for the comparison of the theory with experiment we consider the integral

$$
P\left(x_{c}, t_{c}\right)=\frac{1}{\Gamma_{0}} \iint_{A\left(x_{c}, t_{c}\right)} d x d t \frac{d^{2} \Gamma}{d x d t}
$$

where $x_{c}, t_{c}$ is the point in $(x, t)$ plane sitting not too close to the boundary (outside the resonance range), $\Gamma_{0}=\left|V_{u b}\right|^{2} G_{F}^{2} m_{b}^{5} / 192 \pi^{3}$ and $A\left(x_{c}, t_{c}\right)$ is the area of integration shown on Fig.3 which includes the resonance domain.

The function $P(x, t)$ is plotted as a function of $t$ on Fig.4 for three values of $x$ equal to $0.3,0.6,0.8$. The last value of $x$ is close to the border of the resonance region beyond which we cannot make reliable predictions for the distributions considered. The dashed lines on Fig. 4 describe the leading order distributions in $t$ while the solid lines include QCD corrections we calculated. As we can see it from the curves, the corrections are negative.

\section{Conclusion}

Let us now summarize our results. Model independent approach to nonperturbative effects $\left(1 / m_{q}\right)^{n}$ is used for calculations of differential distributions. The effects are most pronounced near the endpoints of the spectra. We discussed how the comparison with experiment should be formulated accounting for the boundary effects. A somewhat disappointing point is that we cannot use our results to improve an extraction of $V_{u b}$ by the consideration of $q^{2}$ dependence. Indeed, experimentally the signal of $b \rightarrow u$ is due to the range of electron energy $E_{e}$ near the upper end where $b \rightarrow c$ is absent. However as it follows from Fig.3 the distribution in $q^{2}$ at such energies is concentrated in the resonance domain, and no model-independent prediction emerges. 


\section{Acknowledgments}

The authors are grateful to Dr. R. Poling for stimulating discussions of experimentally measured distributions. Those discussions initiated the present study. We also benefitted from helpful discussions with Dr. N. Uraltsev and Dr. M. Voloshin. This work was supported in part by DOE under the grant number DOE-AC02-83ER40105.

After our paper was completed, one of us - B.B. - learned from A. Manohar about the similar work, to be published [19]. 


\section{A Hadronic invariant functions}

Here we present the results of calculations of different hadronic invariant functions $h_{i}$, introduced by eqs.(11, (38)). The structure functions $w_{i}$ are simply related to $h_{i}$ by eqs.(12) and (42). We use the following notation : $q_{0}=q \cdot v, \vec{q}^{2}=q_{0}^{2}-q^{2}$ and $z=m_{Q}^{2}-2 m_{Q} q_{0}+q^{2}-m_{q}^{2}$.

For the Vector $\times$ Vector functions we have:

$$
\begin{gathered}
h_{1}^{V V}=-\left[\left(m_{Q}-m_{q}-q_{0}\right)-\left(\mu_{G}^{2}-\mu_{\pi}^{2}\right) \frac{1}{2 m_{Q}}\left(\frac{1}{3}+\frac{m_{q}}{m_{Q}}\right)\right] \frac{1}{z}- \\
\frac{1}{m_{Q}}\left[\frac{1}{3} \mu_{G}^{2}\left(\left(4 m_{Q}-3 q_{0}\right)\left(m_{Q}-m_{q}-q_{0}\right)+2 \vec{q}^{2}\right)+\right. \\
\left.\mu_{\pi}^{2}\left(q_{0}\left(m_{Q}-m_{q}-q_{0}\right)-\frac{2}{3} \vec{q}^{2}\right)\right] \frac{1}{z^{2}}- \\
\frac{4}{3} \mu_{\pi}^{2} \vec{q}^{2}\left(m_{Q}-m_{q}-q_{0}\right) \frac{1}{z^{3}}, \\
h_{2}^{V V}=-\left[2 m_{Q}-\frac{5}{3 m_{Q}}\left(\mu_{G}^{2}-\mu_{\pi}^{2}\right)\right] \frac{1}{z}- \\
\frac{2}{3}\left[2 \mu_{G}^{2}\left(m_{Q}-m_{q}\right)-5 \mu_{G}^{2} q_{0}+7 \mu_{\pi}^{2} q_{0}\right] \frac{1}{z^{2}}- \\
\frac{8}{3} m_{Q} \mu_{\pi}^{2} \vec{q}^{2} \frac{1}{z^{3}} \\
\left.h_{5}^{V V}=\frac{1}{z}-\frac{1}{3}\left[5 \frac{q_{0}}{m_{Q}}\left(\mu_{G}^{2}-\mu_{\pi}^{2}\right)-4 \mu_{\pi}^{2}\right)\right] \frac{1}{z^{2}}+\frac{4}{3} \mu_{\pi}^{2} \vec{q}^{2} \frac{1}{z^{3}} \\
h_{4}^{V V}=-\frac{4}{3 m_{Q}}\left(\mu_{\pi}^{2}-\mu_{G}^{2}\right) \frac{1}{z^{2}},
\end{gathered}
$$

To get the functions $h_{i}^{A A}$ for Axial $\times$ Axial tensor from $h_{i}^{V V}$ one should substitute $m_{q}$ by $\left(-m_{q}\right)$ in eqs. A.1 - A.5).

For the Axial $\times$ Vector tensor only one invariant structure survives:

$$
h_{3}^{V A}=\frac{1}{z}+\left[2 \mu_{G}^{2}+\frac{5}{3}\left(\mu_{\pi}^{2}-\mu_{G}^{2}\right) \frac{q_{0}}{m_{Q}}\right] \frac{1}{z^{2}}+\frac{4}{3} \mu_{\pi}^{2} \vec{q}^{2} \frac{1}{z^{3}} .
$$


Summing up we get the result for the full hadronic tensor $h_{\mu \nu}$.

$$
\begin{aligned}
& h_{1}=-\left[2\left(m_{Q}-q_{0}\right)-\frac{1}{3 m_{Q}}\left(\mu_{G}^{2}-\mu_{\pi}^{2}\right)\right] \frac{1}{z}- \\
& {\left[\frac{2}{3 m_{Q}} \mu_{G}^{2}\left(4 m_{Q}^{2}+2 \vec{q}^{2}-7 m_{Q} q_{0}+3 q_{0}^{2}\right)+\right.} \\
& \left.\frac{\mu_{\pi}^{2}}{2 m_{Q}}\left(4 q_{0}\left(m_{Q}-q_{0}\right)-\frac{8}{3} \vec{q}^{2}\right)\right] \frac{1}{z^{2}}- \\
& \frac{8}{3} \mu_{\pi}^{2} \vec{q}^{2}\left(m_{Q}-q_{0}\right) \frac{1}{z^{3}} \\
& h_{2}=-\left[4 m_{Q}+\frac{10}{3 m_{Q}}\left(\mu_{\pi}^{2}-\mu_{G}^{2}\right)\right] \frac{1}{z}- \\
& {\left[\frac{28}{3} \mu_{\pi}^{2} q_{0}+\mu_{G}^{2}\left(\frac{8}{3} m_{Q}-\frac{20}{3} q_{0}\right)\right] \frac{1}{z^{2}}-} \\
& \frac{16}{3} \mu_{\pi}^{2} m_{Q} \vec{q}^{2} \frac{1}{z^{3}} \text {. } \\
& h_{3}=-2 \frac{1}{z}-\left[4 \mu_{G}^{2}+\frac{10}{3}\left(\mu_{\pi}^{2}-\mu_{G}^{2}\right) \frac{q_{0}}{m_{Q}}\right] \frac{1}{z^{2}}-\frac{8}{3} \mu_{\pi}^{2} \vec{q}^{2} \frac{1}{z^{3}} \text {, } \\
& h_{4}=-\frac{8}{3 m_{Q}}\left(\mu_{\pi}^{2}-\mu_{G}^{2}\right) \frac{1}{z^{2}}, \\
& \left.h_{5}=2 \frac{1}{z}-\frac{2}{3}\left[5\left(\mu_{G}^{2}-\mu_{\pi}^{2}\right) \frac{q_{0}}{m_{Q}}-4 \mu_{\pi}^{2}\right)\right] \frac{1}{z^{2}}+\frac{8}{3} \mu_{\pi}^{2} \vec{q}^{2} \frac{1}{z^{3}} \text {, }
\end{aligned}
$$




\section{References}

[1] G. Altarelli, N. Cabibbo, G. Corbo, L. Maiani and G. Martinelli, Nucl. Phys., B208 (1982) 365.

[2] N. Isgur, D. Scora, B. Grinstein and M. Wise, Phys. Rev. D39 (1989) 799.

[3] M. Voloshin and M. Shifman, Yad. Fiz. 41 (1985) 187 [Sov. J. Nucl. Phys. 41 (1985) 120]; ZhETF 91 (1986) 1180 [Sov. Phys. JETP 64 (1986) 698].

[4] K. Wilson, Phys. Rev. 179 (1969) 1499;

K. Wilson and J. Kogut, Phys. Rep. 12 (1974) 75.

[5] I. Bigi, N. Uraltsev and A. Vainshtein, Phys. Lett. B293 (1992) 430.

[6] B. Blok and M. Shifman, Nucl. Phys. B399 (1993) 441; 459.

[7] I. Bigi, M. Shifman, N. Uraltsev and A. Vainshtein, "QCD Predictions For Lepton Spectra in Inclusive Heavy Flavor Decays ", March 1992, Preprint TPI-MINN-93/12-T [Phys. Rev. Lett., submitted]. More detailed text is in preparation.

[8] I. Bigi, B. Blok, M. Shifman, N. Uraltsev and A. Vainshtein, "A $Q C D$ 'Manifesto' on Inclusive Decays of Beauty and Charm", Talk at DPF Meeting of APS, November 1992, Preprint TPI-MINN-92/67-T.

[9] J. Chay, H. Georgi and B. Grinstein, Phys. Lett. B247 (1990) 399.

[10] M. Shifman, A. Vainshtein and V. Zakharov, Nucl. Phys. B147 (1979) 385.

[11] J. D. Bjorken, Invited Talk at Les Rencontres de la Valle d'Aosta, La Thuille, 1990, Preprint SLAC-PUB-5278, 1990.

[12] E. Eichten and B. Hill, Phys. Lett. B234 (1990) 511;

H. Georgi, Phys. Lett. B240 (1990) 447.

[13] V. Novikov, M. Shifman, A. Vainshtein and V. Zakharov, Fortsch. Phys. B32 (1984) 585. 
[14] B. Blok and M. Shifman, Nucl. Phys. B389 (1993) 534.

[15] M. Neubert, preprint SLAC-PUB-5770 (1992)

[16] E. Bagan et al., Phys. Lett. B278 (1992) 467

[17] P. Ball et al., Preprint TUM-T31-31-92

[18] R.L.Jaffe and L.Randall, "Heavy Quark Fragmentation Into Heavy Mesons", Preprint CTP \# 2189, May 1993

[19] A. Manohar and M. Wise, In preparation

\section{Figure captions}

Fig.1: The tree diagram determining the transition operator $T_{\mu \nu}$ in the leading approximation. The dashed lines correspond to the weak currents, the solid internal line describes the propagation of the quark $q$ and the bold external lines represent the heavy quark $Q$.

Fig.2: The tree diagram determining the operator without the heavy quark $Q$. Now the bold internal line describes the propagation of the heavy quark $Q$ and the solid external lines represent the quark $q$.

Fig.3: The kinematical region of the decay for $b \rightarrow u$ decays in coordinates $x=2 E_{e} / m_{b}$ and $t=q^{2} / 2 m_{b} E_{e}$. The solid lines are the kinematical boundary for the $b$ quark decay $\left(x_{\max }=t_{\max }=1\right)$ and the dashed lines are the boundary for $B$ meson decay $\left(x_{\max }=t_{\max }=M_{B} / m_{b}\right)$. The area of integration for the distribution $P(x, t)$ (see eq.(55)) is shadowed. It includes integration over the resonance domain.

Fig.4: The integrated distribution $P(x, t)$ (see eq.(55) ) for the case $b \rightarrow u$ is plotted as function of $t=q^{2} / 2 m_{b} E_{e}$ for few values of $x=2 E_{e} / m_{b}$. The dashed lines correspond to the leading order distribution while the solid lines account for QCD corrections. The lines stop at the border of resonance region. The corrections are negative and their relative magnitude becomes larger when $x$ approaches the resonance region. 